PROCEEDINGS OF THE

AMERICAN MATHEMATICAL SOCIETY

Volume 131, Number 12, Pages 3665-3672

S 0002-9939(03)06938-7

Article electronically published on February 28, 2003

\title{
COTILTING MODULES ARE PURE-INJECTIVE
}

\author{
S. BAZZONI
}

(Communicated by Martin Lorenz)

\begin{abstract}
We prove that a cotilting module over an arbitrary ring is pureinjective.
\end{abstract}

Tilting (and cotilting) modules were first defined in the case of finite-dimensional algebras by Brenner and Butler [5] and by Happel and Ringel [19]. Generalizations over arbitrary rings have been considered by many authors: Colby and Fuller [7], Colpi [8] and Miyashita 23. In [10] Colpi and Trlifaj defined the notion of tilting modules in the case of infinitely generated modules and in [1] Angeleri, Tonolo and Trlifaj proved that tilting and cotilting classes provide for special preenvelopes and precovers generalizing the analogous results proved by Auslander, Reiten and Smalø 2, 3] about left and right approximations.

The definitions of tilting and cotilting infinitely generated modules involve the notions of torsion and cotorsion theories. While the first one is a classical notion and widely investigated, the second one, introduced by Salce 24] in the case of abelian groups, was ignored for 20 years, until, very recently, its importance was noted by Göbel and Shelah [16, 15] in connection with the problem of finding non-trivial splitter groups. Moreover, it turned out to be a useful tool in proving the Flat Cover Conjecture [4]. In fact, Enochs's proof of this conjecture makes use of the notion of cotorsion theory together with the very powerful result proved by Eklof and Trlifaj [12], stating that cotorsion theories cogenerated by a set of modules are complete, i.e., they provide for special preenvelopes and special precovers (see Section 3 for definitions).

The definition of a cotilting module valid over an arbitrary ring $R$ states that an $R$-module $U$ is a cotilting if ${ }^{\perp} U=\operatorname{Cogen} U$, where ${ }^{\perp} U=\{M \in R$-Mod $\left.\operatorname{Ext}_{R}^{1}(M, U)=0\right\}$ and Cogen $U$ is the class of $R$-modules cogenerated by $U$, namely the class of modules which are embeddable in a direct product of copies of $U$. Another way of putting this is to say that $U$ is cotilting if the cotorsion-free class of the cotorsion theory generated by $U$ coincides with Cogen $U$ (see Section 1). In [13] Eklof and Trlifaj proved that a cotorsion theory generated by a class of pure-injective modules is complete and, as noted above, a cotilting torsion-free class provides for preenvelopes and precovers. Thus the question raised by Trlifaj of whether a cotilting module is pure-injective is particularly interesting. In this

Received by the editors May 31, 2002 and, in revised form, July 10, 2002.

1991 Mathematics Subject Classification. Primary 16D90, 16D30.

Key words and phrases. Cotilting modules, pure-injective modules, cotorsion theories.

This research was supported by MURST. 
note we answer the question affirmatively, namely we prove that if $U$ is a cotilting module over any ring $R$, then $U$ is a pure-injective module.

\section{Preliminaries}

$R$ will denote an associative ring with identity and $R$-Mod the class of left $R$ modules. Recall that a torsion theory is a pair $(\mathcal{T}, \mathcal{F})$ of classes of modules which are mutually orthogonal with respect to the $\operatorname{Hom}_{R}$ functor, i.e., such that

$$
\begin{aligned}
& \mathcal{T}=\left\{T \in R \text { - Mod } \mid \operatorname{Hom}_{R}(T, F)=0 \text { for all } F \in \mathcal{F}\right\}, \\
& \mathcal{F}=\left\{F \in R \text {-Mod } \mid \operatorname{Hom}_{R}(T, F)=0 \text { for all } T \in \mathcal{T}\right\} .
\end{aligned}
$$

$\mathcal{T}$ is called a torsion class and its objects are called torsion modules; $\mathcal{F}$ is called a torsion-free class and its objects are called torsion-free modules. They can both be characterized in terms of closure properties: $\mathcal{T}$ is a torsion class if and only if it is closed under epimorphic images, direct sums and extensions; while $\mathcal{F}$ is a torsion-free class if and only if it is closed under submodules, direct products and extensions. Given a class $\mathcal{M}$ of modules, the torsion theory generated by $\mathcal{M}$ is the smallest torsion theory for which the objects of $\mathcal{M}$ are torsion. Dually, the torsion theory cogenerated by $\mathcal{M}$ is the smallest torsion theory for which the objects of $\mathcal{M}$ are torsion-free. For more details, see [25] Ch. VI].

Considering the functor $\mathrm{Ext}_{R}^{1}$ instead of the functor $\mathrm{Hom}_{R}$, Salce in [24] introduced the notion of a cotorsion theory.

A cotorsion theory is a pair $(\mathcal{A}, \mathcal{B})$ of classes of modules over a ring $R$ which are mutually orthogonal with respect to the $\operatorname{Ext}_{R}^{1}$ functor, i.e., such that

$$
\begin{aligned}
& \mathcal{A}={ }^{\perp} \mathcal{B}=\left\{A \in R \text { - Mod } \mid \operatorname{Ext}_{R}^{1}(A, B)=0 \text { for all } B \in \mathcal{B}\right\}, \\
& \mathcal{B}=\mathcal{A}^{\perp}=\left\{B \in R \text {-Mod } \mid \operatorname{Ext}_{R}^{1}(A, B)=0 \text { for all } A \in \mathcal{A}\right\} .
\end{aligned}
$$

$\mathcal{A}$ is called the cotorsion-free class, while $\mathcal{B}$ is called the cotorsion class. Given a class $\mathcal{M}$ of modules, the pairs

$$
\mathcal{G}_{\mathcal{M}}=\left({ }^{\perp} \mathcal{M},\left({ }^{\perp} \mathcal{M}\right)^{\perp}\right) \quad \text { and } \quad \mathcal{C}_{\mathcal{M}}=\left({ }^{\perp}\left(\mathcal{M}^{\perp}\right), \mathcal{M}^{\perp}\right)
$$

are cotorsion theories, called the cotorsion theories generated and cogenerated by $\mathcal{M}$, respectively.

For every $R$-module $M$, Prod $M$ will denote the class of modules isomorphic to summands of direct products of copies of $M$ and $\operatorname{Cogen} M$ will denote the class of the $R$-modules cogenerated by $M$, namely the class of modules which are embeddable in a product of copies of $M$. It is evident that an $R$-module $N \in$ Cogen $M$ if and only if, for every $0 \neq x \in N$, there is a morphism $f \in \operatorname{Hom}_{R}(N, M)$ such that $f(x) \neq 0$.

Definition 1. If $R$ is any ring, an $R$-module $U$ is said to be cotilting if ${ }^{\perp} U=$ Cogen $U$.

If $U$ is a cotilting module, then ${ }^{\perp} U=\operatorname{Cogen} U$ is the torsion-free class of the torsion theory cogenerated by $U$; moreover it is the cotorsion-free class of the cotorsion theory generated by $U$. A torsion-free class $\mathcal{F}$ is called a cotilting torsion-free class if $\mathcal{F}={ }^{\perp} U$ for some cotilting module $U$. 
The above definition of a cotilting module generalizes the classical notion. In fact, as proved in [1] and [9], an $R$-module $U$ is cotilting if and only if the following three conditions hold:

1. i.d. $U \leq 1$;

2. $\operatorname{Ext}_{R}^{1}\left(U^{\lambda}, U\right)=0$ for every cardinal $\lambda$;

3 . there exists an exact sequence

$$
0 \rightarrow U_{1} \rightarrow U_{0} \rightarrow E \rightarrow 0
$$

where $E$ is an injective cogenerator of $R$ - Mod and $U_{0}, U_{1} \in \operatorname{Prod} U$.

Moreover, $U$ is cotilting if and only if $U$ satisfies 1,2 and

$3^{\prime}$. for any $R$ module $M, \operatorname{Hom}_{R}(M, U)=0$ and $\operatorname{Ext}_{R}^{1}(M, U)=0$ imply $M=0$.

Definition 2. If $R$ is any ring, an $R$-module $U$ is said to be partial cotilting if $U$ satisfies 1 and 2 above, i.e., if i.d. $U \leq 1$ and Cogen $U \subseteq{ }^{\perp} U$.

Note that for any $R$-module $M$, i.d. $M \leq 1$ if and only if ${ }^{\perp} M$ is closed under submodules; moreover if i.d. $M \leq 1$, then ${ }^{\perp} M$ is a torsion-free class if and only if it is closed under direct products. In [9], a module $U$ is said to be partial cotilting if Cogen $U \subseteq{ }^{\perp} U$ and ${ }^{\perp} U$ is a torsion-free class: this notion is then stronger than the one given by Definition 2 .

The notion of pure-injectivity will play a central role in our discussion. We refer to Theorem 7.1 in $[18$ for a complete description of the various equivalent conditions satisfied by pure-injective modules.

\section{Cotilting MOdules AND PURE-INJECTIVITY}

The characterization of pure-injectivity that will be particularly useful for our purposes is condition (vi) in [18, Theorem 7.1]. We state it explicitly.

Lemma 2.1. An R-module $M$ is pure-injective if and only if, for every cardinal $\gamma$, every homomorphism $f: M^{(\gamma)} \rightarrow M$ extends to a homomorphism $g: M^{\gamma} \rightarrow M$.

Thus, in particular, we obtain:

Corollary 2.2. Let $M$ be an $R$-module such that $\operatorname{Ext}_{R}^{1}\left(M^{\gamma}, M\right)=0$, for every cardinal $\gamma$. Then $M$ is pure-injective if and only if $\operatorname{Ext}_{R}^{1}\left(\frac{M^{\gamma}}{M^{(\gamma)}}, M\right)=0$, for every cardinal $\gamma$.

Proof. Consider the exact sequence

$$
0 \rightarrow M^{(\gamma)} \rightarrow M^{\gamma} \rightarrow \frac{M^{\gamma}}{M^{(\gamma)}} \rightarrow 0
$$

Applying the contravariant functor $\operatorname{Hom}_{R}(-, M)$, we obtain the exact sequence

$$
\operatorname{Hom}_{R}\left(M^{\gamma}, M\right) \rightarrow \operatorname{Hom}_{R}\left(M^{(\gamma)}, M\right) \rightarrow \operatorname{Ext}_{R}^{1}\left(\frac{M^{\gamma}}{M^{(\gamma)}}, M\right) \rightarrow 0 .
$$

By the preceding lemma we get the conclusion.

If $X$ is a set, we say that a family $\mathcal{A}$ of subsets of $X$ is almost disjoint if the intersection of any two distinct elements of $\mathcal{A}$ is finite. We now need some cardinal arguments. The following fact is well known in the literature and it has many different proofs. The one we prefer is the following.

Lemma 2.3. Let $\lambda$ be any infinite cardinal. Then there is a family of $\lambda^{\aleph_{0}}$ countable almost disjoint subsets of $\lambda$. 
Proof. Consider the tree $T$ of the finite sequences of elements of $\lambda$, i.e., $T=\{t: n \rightarrow$ $\lambda \mid n \in \omega\}$. Clearly $T=\bigcup_{n \in \omega} \lambda^{n}$, hence $|T|=\lambda$. For every function $f: \omega \rightarrow \lambda$, consider the subset $A_{f}$ of $T$ defined by $A_{f}=\left\{f \Gamma_{n}: n \in \omega\right\} . A_{f}$ is nothing else than an $\omega$-branch of $T$. Clearly, if $f, g$ are two different functions in $\lambda^{\omega}, A_{f} \cap A_{g}$ is finite. Thus $\left\{A_{f}\right\}_{f \in \lambda^{\aleph_{0}}}$ is a family of $\lambda^{\aleph_{0}}$ countable almost disjoint subsets of $T$. Considering a bijection of $\lambda$ to $T$, we have the desired conclusion.

The following result is also well known (see for instance [17, Lemma 3.1]).

Lemma 2.4. For any cardinal $\nu$ there is a cardinal $\lambda \geq \nu$ such that $\lambda^{\aleph_{0}}=2^{\lambda}$.

Using the preceding results and a cardinal argument similar to the one used by Hunter in [20], we can prove the following.

Proposition 2.5. Let $U$ be an $R$-module such that ${ }^{\perp} U$ is closed under direct products and submodules. Then $\operatorname{Ext}_{R}^{1}\left(\frac{M^{\aleph_{0}}}{M^{\left(\aleph_{0}\right)}}, U\right)=0$, for any $R$-module $M$ such that $\operatorname{Ext}_{R}^{1}(M, U)=0$.

Proof. Let $X=\frac{M^{\aleph_{0}}}{M^{\left(\aleph_{0}\right)}}$. Consider an infinite cardinal $\lambda$ and let $\left\{A_{\beta}\right\}_{\beta \in \lambda^{\aleph_{0}}}$ be a family of $\lambda^{\aleph_{0}}$ countable almost disjoint subsets of $\lambda$. View $M^{A_{\beta}}$ as embedded in $M^{\lambda}$ and let $\eta_{\beta}: M^{A_{\beta}} \rightarrow \frac{M^{\lambda}}{M^{(\lambda)}}$ be the restriction to $M^{A_{\beta}}$ of the canonical projection of $M^{\lambda}$ onto $\frac{M^{\lambda}}{M^{(\lambda)}}$; then $\operatorname{Ker} \eta_{\beta}=M^{\left(A_{\beta}\right)}$ and $\operatorname{Im} \eta_{\beta} \cong X$. Since the sets $\left\{A_{\beta}\right\}$ are pairwise almost disjoint, the $\operatorname{sum} \sum_{\beta} \operatorname{Im} \eta_{\beta}$ in $\frac{M^{\lambda}}{M^{(\lambda)}}$ is a direct sum.

In fact, assume $\sum_{i=1}^{n} \eta_{\beta_{i}}\left(x_{\beta_{i}}\right)=0$, for some $x_{\beta_{i}} \in M^{A_{\beta_{i}}}(i=1, \ldots, n)$. Let $y=\sum_{i=1}^{n} x_{\beta_{i}}$; then $y \in M^{(\lambda)}$. Let $F$ be the support of $y$ and $G=\bigcup_{i \neq j}\left(A_{\beta_{i}} \cap A_{\beta_{j}}\right)$; then $F \cup G$ is finite. Fix an index $i \in\{1, \ldots, n\}$; for every $\alpha \in A_{\beta_{i}} \backslash(G \cup F)$, the $\alpha$-component $y(\alpha)$ of $y$ is 0 , hence $x_{\beta_{i}}(\alpha)=0$ too, since $\alpha \notin A_{\beta_{j}}$, for every $j \neq i$. Thus $x_{\beta_{i}} \in M^{(\lambda)}$; hence $\eta_{\beta_{i}}\left(x_{\beta_{i}}\right)=0$ for every $i$.

Let $V$ be the submodule of $M^{\lambda}$ such that $\frac{V}{M^{(\lambda)}} \cong \bigoplus_{\beta \in \lambda^{\aleph_{0}}} \operatorname{Im} \eta_{\beta} \cong X^{\left(\lambda^{\aleph_{0}}\right)}$. By Lemma 2.4 we can choose $\lambda$ such that $\lambda \geq\left|\operatorname{Hom}_{R}(M, U)\right|$ and $\lambda^{\aleph_{0}}=2^{\lambda}$. Since ${ }^{\perp} U$ is closed under products and submodules, we have that $\operatorname{Ext}_{R}^{1}(V, U)=0$. The exact sequence

$$
0 \rightarrow M^{(\lambda)} \rightarrow V \rightarrow X^{\left(\lambda^{\aleph_{0}}\right)} \rightarrow 0
$$

induces the exact sequence

$$
\operatorname{Hom}_{R}\left(M^{(\lambda)}, U\right) \rightarrow \operatorname{Ext}_{R}^{1}\left(X^{\left(\lambda^{\aleph_{0}}\right)}, U\right) \rightarrow 0 .
$$

The cardinality of the first term is $\left|\operatorname{Hom}_{R}(M, U)\right|^{\lambda}$ which coincides with $2^{\lambda}$ by the assumption $\lambda \geq\left|\operatorname{Hom}_{R}(M, U)\right|$. The term $\operatorname{Ext}_{R}^{1}\left(X^{\left(\lambda^{\aleph_{0}}\right)}, U\right)$ is isomorphic to $\prod_{\lambda^{\aleph_{0}}} \operatorname{Ext}_{R}^{1}(X, U)$. Thus, if $\operatorname{Ext}_{R}^{1}(X, U)$ is non-zero, the cardinality of the second term in the above sequence is at least $2^{\lambda^{\aleph_{0}}}=2^{2^{\lambda}}$, contradicting the existence of an epimorphism.

Corollary 2.6. Let $R$ be any ring and $U$ a partial cotilting $R$-module. Then $\operatorname{Ext}_{R}^{1}\left(\frac{U^{\aleph_{0}}}{U^{\left(\aleph_{0}\right)}}, U\right)=0$. In particular, if $U$ is cotilting, then $\frac{U^{\aleph_{0}}}{U^{\left(\aleph_{0}\right)}} \in \operatorname{Cogen} U$.

Proof. Let $M=U$ in the proof of the preceding proposition. Then the proof is valid under the hypothesis that $U$ is partial cotilting. The last statement follows immediately from the definition of a cotilting module. 
The following result is valid in all generality. It will be a key step in proving the main theorem.

Lemma 2.7. Let $M, U$ be $R$-modules. If $\frac{M^{\aleph_{0}}}{M^{\left(\aleph_{0}\right)}} \in \operatorname{Cogen} U$, then $\frac{M^{\gamma}}{M^{(\gamma)}} \in \operatorname{Cogen} U$, for any cardinal $\gamma$.

Proof. We show that for every $0 \neq x \in \frac{M^{\gamma}}{M^{(\gamma)}}$ there is a map $f \in \operatorname{Hom}_{R}\left(\frac{M^{\gamma}}{M^{(\gamma)}}, U\right)$ such that $f(x) \neq 0$. We can write $x=\left(y_{\alpha}\right)_{\alpha \in \gamma}+M^{(\gamma)}$, where $y=\left(y_{\alpha}\right)_{\alpha \in \gamma} \in M^{\gamma}$ is an element with infinite support. Choose a subset $A$ of the support of $y$ such that $|A|=\aleph_{0}$ and consider the projection $\pi_{A}: M^{\gamma} \rightarrow M^{A}, \pi_{A}\left(\left(z_{\alpha}\right)_{\alpha \in \gamma}\right)=\left(z_{\alpha}\right)_{\alpha \in A}$. Let $\rho$ denote the canonical epimorphism $\rho: M^{A} \rightarrow \frac{M^{A}}{M^{(A)}}$. The kernel of the composition $\rho \circ \pi_{A}$ contains $M^{(\gamma)}$, thus $\rho \circ \pi_{A}$ factors through a map $g: \frac{M^{\gamma}}{M^{(\gamma)}} \rightarrow \frac{M^{A}}{M^{(A)}}$. Clearly $g(x)=\left(y_{\alpha}\right)_{\alpha \in A}+M^{(A)}$, thus $g(x) \neq 0$, since $A$ was an infinite subset of the support of $y$. By the assumption $\frac{M^{\aleph_{0}}}{M^{\left(\aleph_{0}\right)}} \in \operatorname{Cogen} U$ we can find a morphism $h: \frac{M^{\aleph_{0}}}{M^{\left(\aleph_{0}\right)}} \rightarrow U$ such that $h(g(x)) \neq 0$; hence $h \circ g$ is the wanted map $f$.

We are now in a position to prove our main result.

Theorem 2.8. Let $U$ be a partial cotilting $R$-module such that ${ }^{\perp} U$ is closed under direct products. Then $U$ is pure-injective. In particular, if $U$ is cotilting, then $U$ is pure-injective.

Proof. By [9, Theorem 2.11] a module satisfying our hypotheses is a direct summand of a cotilting module. Thus we may assume that $U$ is cotilting. By Corollary 2.2 $U$ is pure-injective if and only if $\frac{U^{\gamma}}{U^{(\gamma)}} \in{ }^{\perp} U$, for every cardinal $\gamma$, i.e., if and only if $\frac{U^{\gamma}}{U(\gamma)} \in$ Cogen $U$. By Lemma 2.7 it is enough to consider the case $\gamma=\omega$. Thus the conclusion follows by Corollary 2.6.

\section{Applichtions}

In [14], Enochs introduced the notions of $\mathcal{X}$-precover and of $\mathcal{X}$-cover, for any class $\mathcal{X}$ of modules and in [26] the notion of a special $\mathcal{X}$-precover was defined. They generalize the analogous definitions of right and left approximations considered by Auslander, Reiten and Smalø [2] 3]. We now recall the definitions.

Definition 3. Let $\mathcal{X}$ be a class of modules, $M$ an $R$-module and $X \in \mathcal{X}$. A homomorphism $\phi \in \operatorname{Hom}_{R}(X, M)$, is called an $\mathcal{X}$-precover of $M$ if for every homomorphism $\phi^{\prime} \in \operatorname{Hom}_{R}\left(X^{\prime}, M\right)$ with $X^{\prime} \in \mathcal{X}$ there exists a homomorphism $f: X^{\prime} \rightarrow X$ such that $\phi^{\prime}=\phi f$. An $\mathcal{X}$-precover, $\phi \in \operatorname{Hom}_{R}(X, M)$, is called an $\mathcal{X}$-cover of $M$ if for every endomorphism $f$ of $X$ such that $\phi=\phi f, f$ is an automorphism of $X$. An $\mathcal{X}$-precover $\phi$ of $A$ is said to be special if $\phi$ is surjective and $\operatorname{Ker} \phi \in \mathcal{X}^{\perp}$.

The notions of $\mathcal{X}$-preenvelope, special $\mathcal{X}$-preenvelope and $\mathcal{X}$-envelope are defined dually.

The class $\mathcal{X}$ is said to be a precover class (cover class, preenvelope class, envelope class $)$ if every $R$-module admits an $\mathcal{X}$-precover $(\mathcal{X}$-cover, $\mathcal{X}$-preenvelope, $\mathcal{X}$-envelope).

If $(\mathcal{A}, \mathcal{B})$ is a cotorsion theory, then every $R$-module admits a special $\mathcal{A}$-precover if and only if every $R$-module admits a special $\mathcal{B}$-preenvelope (see [24. Corollary $2.4])$. The cotorsion theory $(\mathcal{A}, \mathcal{B})$ is said to be complete if every $R$-module admits a special $\mathcal{A}$-precover, while it is said to be perfect if $\mathcal{A}$ is a cover class and $\mathcal{B}$ is an envelope class. In [12] Theorem 10] it is shown that a cotorsion theory cogenerated 
by a set of modules is complete; moreover, as a consequence of [13, Corollary 10] it follows that the cotorsion theory generated by a class of pure-injective modules is perfect. Thus, as a corollary of Theorem [2.8, we obtain that if $U$ is a cotilting $R$ module, then the cotorsion theory generated by $U$ is perfect; in particular $\operatorname{Cogen} U$ is a cover class. We could get the same conclusion by using a result proved in [1]. In fact, we can state the following.

Proposition 3.1. Let $\mathcal{F}$ be a class of modules closed under products and submodules. The following conditions are equivalent:

1. $\mathcal{F}$ is a cotilting torsion-free class;

2. every module has a special $\mathcal{F}$-precover;

3. $\mathcal{F}$ is a cover class containing all projective modules and closed under extensions.

Proof. The equivalence $1 \Leftrightarrow 2$ is proved in [1, Theorem 2.5].

$1 \Rightarrow 3$. Assume $\mathcal{F}$ is a cotilting torsion-free class; then by Theorem 2.8, $\mathcal{F}$ is closed under direct limits. In fact, let $U$ be a cotilting module such that $\mathcal{F}={ }^{\perp} U$ and let $\left\{N_{i}\right\}_{i \in I}$ be a direct system of modules in $\mathcal{F}$. The exact sequence

$$
0 \rightarrow B \rightarrow \bigoplus_{i} N_{i} \rightarrow \lim _{\rightarrow} N_{i} \rightarrow 0
$$

is pure exact. We have $\operatorname{Ext}_{R}^{1}\left(\bigoplus_{i} N_{i}, U\right)=0$, since $N_{i} \in \mathcal{F}$. Thus, $\operatorname{Ext}_{R}^{1}\left(\lim _{\rightarrow} N_{i}, U\right)$ $=0$, since $U$ is pure-injective. By [26. Theorem 2.2.8] and by the implication $1 \Rightarrow 2$, $\mathcal{F}$ is a cover class. Clearly $\mathcal{F}$ contains the projective modules and is closed under extensions.

$3 \Rightarrow 2$. By the hypotheses and by [26] Lemma 2.1], any $\mathcal{F}$-cover is a special $\mathcal{F}$-precover.

Remark 1. In 22 it is proved that a cotilting module is pure-injective if and only if ${ }^{\perp} U$ is closed under direct limits. The necessity is obtained arguing in the same way as in the proof of the preceding proposition. For the other implication an alternative easy proof could be given by noting that $\frac{U^{\gamma}}{U^{(\gamma)}}$ is isomorphic to the direct limit $\lim _{\rightarrow} \frac{U^{\gamma}}{U^{F}}$ where $F$ vary among the finite subsets of $\gamma$. Thus, $\frac{U^{\gamma}}{U^{(\gamma)}} \in{ }^{\perp} U$, since it is a direct limit of products of copies of $U$ and the conclusion follows by Corollary 2.2.

Theorem 2.8 can also be applied to obtain other interesting properties of the class ${ }^{\perp} U$, for $U$ a cotilting $R$-module. Recall that a class $\mathcal{X}$ of $R$-modules is said to be definable if there exists a family of coherent functors $\left\{F_{i}\right\}_{i \in I}$ such that an $R$-module $M$ is in $\mathcal{X}$ if and only if $F_{i}(M)=0$ for every $i \in I$. In [11] it is proved that a class $\mathcal{X}$ is definable if and only if $\mathcal{X}$ is closed under direct limits, pure submodules and direct products. If $U$ is a cotilting $R$-module, then ${ }^{\perp} U$ is clearly a torsion-free class and, as a consequence of Theorem 2.8, we obtain that it is also closed under direct limits; so it is a definable class. Moreover, in $[6$ it is shown that the equivalence classes of pure-injective cotilting modules form a set, thus now we have that this result holds without the assumption of pure-injectivity. The next proposition summarizes the above observations.

Proposition 3.2. Let $U$ be a cotilting module over a associative ring $R$. Then ${ }^{\perp} U$ is a definable class and the equivalence classes of $R$-cotilting modules form a set. 


\section{ACKNOWLEDGEMENTS}

We thank Riccardo Colpi for the frequent and useful conversations on the subject and, in particular, for suggesting the use of Corollary 2.2 as a criterion to prove pure-injectivity. We also thank Jan Trlifaj for pointing out that the hypothesis in our original version of Theorem 2.8 could be weakened.

\section{REFERENCES}

1. L. Angeleri Hügel, A. Tonolo, J. Trlifaj, Tilting preenvelopes and cotilting precovers, Algebr. Represent. Theory 4 (2001), 155-170. MR 2002e:16010

2. M. Auslander, I. Reiten, Applications of contravariantly finite subcategories, Adv. Math. 86 (1991), 111-152. MR 92e:16009

3. M. Auslander, S. Smalø, Preprojective modules over Artin algebras, J. Algebra 66 (1980), 61-122. MR 83a:16039

4. L. Bican, R. El Bashir and E. Enochs, All modules have flat covers, Bull. London Math. Soc. 33, no. 4 (2001), 385-390. MR 2002e:16002

5. S. Brenner, M. Butler, Generalizations of the Bernstein-Gelfand-Ponomarev reflection functors, in Proc. ICRA III LNM 832, Springer (1980), 103-169. MR 83e:16031

6. A. B. Buan, H. Krause, $\varnothing$. Solberg, On the lattice of cotilting modules, AMA Algebra Montp. Announc., 1 (2002), 6pp.

7. R. R. Colby, K. R. Fuller, Tilting, cotilting and serially tilted rings, Comm. Algebra 18(5) (1990), 1585-1615. MR 91h:16011

8. R. Colpi, Tilting modules and *-modules, Comm. Algebra 21 (1993), 1095-1102. MR 94d:16009

9. R. Colpi, A. Tonolo, J. Trlifaj, Partial cotilting modules and the lattices induced by them, Comm. Algebra 25(10) (1997), 3225-3237. MR 98i:16003

10. R. Colpi, J. Trlifaj, Tilting modules and tilting torsion theories, J. Algebra 178 (1995), 492510. MR 97e: 16003

11. W. W. Crawley-Boevey, Infinite-dimensional modules in the representation theory of finitedimensional algebras, Algebras and modules, I (Trondheim, 1996), 29-54. MR 99m:16016

12. P. C. Eklof and J. Trlifaj, How to make Ext vanish, Bull. London Math. Soc. 33 (2001), 41-51. MR 2001i:16015

13. P. C. Eklof, J. Trlifaj, Covers induced by Ext, J. Algebra 231 (2000), 640-651. MR 2001f:16021

14. E. Enochs, Injective and flat covers, envelopes and resolvents, Israel J. Math. 39 (1981), 33-38. MR 83a:16031

15. R. Göbel, S. Shelah, Almost free splitters, Colloq. Math. 81 (1999), no. 2, 193-221. MR 2000m:20092

16. R. Göbel, S. Shelah, Cotorsion theories and splitters, Trans. Amer. Math.Soc. 352 (2000), no. 11, 5357-5379. MR 2001b:20098

17. P. Griffith, On a subfunctor of Ext, Arch. Math. XXI (1970), 17-22. MR 41:6964

18. C. U. Jensen, H. Lenzing, Model Theoretic Algebra, Gordon and Breach S. Publishers, (1989). MR 91m:03038

19. D. Happel, C. Ringel, Tilted algebras, Trans. Amer. Math. Soc. 274 (1982), 399-443. MR 84d:16027

20. R. H. Hunter, Balanced subgroups of abelian groups, Trans. Amer. Math. Soc. 215 (1976), 81-98. MR 58:22337

21. H. Krause, Ø. Solberg, Filtering modules of finite projective dimension, to appear in Forum Math.

22. F. Mantese, P. Růžička, A. Tonolo, Cotilting versus pure-injective modules, to appear in Pacific J. Math.

23. Y. Miyashita, Tilting modules of finite projective dimension, Math. Z. 193 (1986), 113-146. MR 87m:16055

24. L. Salce, Cotorsion theories for abelian groups, Symposia Math., XXIII (1979), 11-32. MR 81j:20078 
25. B. Stenström, Rings of quotients, GTM, 131, Springer-Verlag, New York (1975). MR 52:10782

26. J. Xu, Flat covers of modules, Lecture Notes in Mathematics No. 1634, Springer-Verlag, New York (1996). MR 98b:16003

Dipartimento di Matematica Pura e Applicata, Università di Padova, Via Belzoni 7 , 35131 PADOVA, ITALY

E-mail address: bazzoni@math.unipd.it 